\title{
Mesenchymal stem cells as a valuable agent in osteoarthritis treatment
}

\author{
Mohsen Sheykhhasan ${ }^{1,2}$, Hamed Manoochehri ${ }^{1}$, Mona Pourjafar ${ }^{1}$, Nashmin Fayazi ${ }^{1}$ \\ ${ }^{1}$ Research Center for Molecular Medicine, Hamadan University of Medical Sciences, Hamadan, Iran; ${ }^{2}$ Department of Mesenchymal Stem Cell, the \\ Academic Center for Education, Culture and Research, Qom Branch, Qom, Iran \\ Correspondence to: Mohsen Sheykhhasan. Research Center for Molecular Medicine, Hamadan University of Medical Sciences, Hamadan, Iran. \\ Email: mohsen.sh2009@gmail.com.
}

Received: 28 July 2018; Accepted: 11 November 2018; Published: 21 November 2018.

doi: $10.21037 /$ sci.2018.11.04

View this article at: http://dx.doi.org/10.21037/sci.2018.11.04

Osteoarthritis (OA), a common disease that originates from cartilage damage, affects approximately 250 million people around the world (1). The disease is more common in older people. Among younger and middle-aged people, injuries are the most common causative agents for $\mathrm{OA}(2)$. The application of conventional treatment methods, such as pain medication and physical therapy techniques, could temporarily alleviate pain, but these methods do not have the ability to recover the primary function of the cartilage (3). Additionally, many difficulties are encountered in autologous chondrocyte implantation for cartilage regeneration, such as donor site damage and cell limited life span after transplantation $(4,5)$. These problems make new therapeutic methods, such as regenerative medicine, to repair the damaged cartilage tissue an exciting prospect (6).

Mesenchymal stem cells (MSCs), as a suitable cell source, are considered as novel therapeutic agents for cartilage and bone diseases and injuries because of their special ability to differentiate into a variety of cell types and their potent capability to self-renewal and repair, as well as their high healing ability and their growth factor secretory capacity (6). MSCs have been identified in many tissues, including bone marrow, adipose tissue, and synovial tissue (6). In addition, because lack of human leucocyte antigen (HLA) class II expression, mesenchymal stem cells are also able to retain immunomodulatory activity in vivo (7). As a result, both autologous and allogeneic MSCs could act as hope agents in OA treatment because of their immunomodulatory properties.

Preclinical studies have confirmed the success of treatment using MSCs on animal models with OA $(8,9)$. According to a review article presented by Xing et al., of
23 studies performed on animals using MSCs through 2017, 21 showed promising results (10). Additionally, an MSCbased therapy has been assessed as a viable alternative for treating cartilage-bone disease, particularly OA (11). MSCs have even been used in many clinical trials in different phases for curing cartilage defects in patients with $\mathrm{OA}$; one review study found that MSC-based therapy has been used to treat $\mathrm{OA}$ in 18 clinical trials (12).

Apart from MSCs, exosomes from MSCs have a beneficial role in OA treatment too.

Exosomes released by MSCs, not only have a promoted cartilage defect regeneration activity via shuttle bioactive RNAs and growth factors associated with different cellular and biological process, i.e., cell proliferation, differentiation, and tissue reconstruction between cells, but are also related to accelerate subchondral bone restoration, which has been demonstrated as an innovative target for OA drug delivery strategy (13-15).

Taken together, the preclinical and clinical studies' results strongly suggest that MSCs and exosomes from MSCs may play a curative role in $\mathrm{OA}$, which brings increased interest in their potential for therapeutic application.

\section{Acknowledgements}

None.

\section{Footnote}

Conflicts of Interest: The authors have no conflicts of interest to declare. 


\section{References}

1. Lim SS, Vos T, Flaxman AD, et al. A comparative risk assessment of burden of disease and injury attributable to 67 risk factors and risk factor clusters in 21 regions, 1990-2010: a systematic analysis for the Global Burden of Disease Study 2010. Lancet 2012;380:2224-60.

2. Felson DT. Osteoarthritis as a disease of mechanics. Osteoarthritis Cartilage 2013;21:10-5.

3. Ivirico JLE, Bhattacharjee M, Kuyinu E, et al. Regenerative engineering for knee osteoarthritis treatment: biomaterials and cell-based technologies. Engineering 2017;3:16-27.

4. Sheykhhasan M, Qomi RT, Kalhor N, et al. Evaluation of the ability of natural and synthetic scaffolds in providing an appropriate environment for growth and chondrogenic differentiation of adipose-derived mesenchymal stem cells. Indian J Orthop 2015;49:561-8.

5. Sheykhhasan M, Ghiasi M, Pak HB. The assessment of natural scaffolds ability in chondrogenic differentiation of human adipose-derived mesenchymal stem cells. Internet Journal of Medical Update 2016;11:11-6.

6. Sheykhhasan M. Mesenchymal Stem Cells and Platelet Derived Concentrates in Regenerative Medicine. J Stem Cell Res Ther 2017;2:00079.

7. Gao F, Chiu S, Motan D, et al. Mesenchymal stem cells and immunomodulation: current status and future prospects. Cell Death Dis 2016;7:e2062.

8. McKinney JM. Intra-articular delivery of encapsulated

doi: $10.21037 /$ sci.2018.11.04

Cite this article as: Sheykhhasan M, Manoochehri H, Pourjafar M, Fayazi N. Mesenchymal stem cells as a valuable agent in osteoarthritis treatment. Stem Cell Investig 2018;5:41. human mesenchymal stem cells reduces osteoarthritis progression in a rat model 2017. Available online: https:// open.bu.edu/handle/2144/23714

9. Wu KC, Chang YH, Liu HW, et al. Transplanting human umbilical cord mesenchymal stem cells and hyaluronate hydrogel repairs cartilage of osteoarthritis in the minipig model. Tzu Chi Med J 2018. [Epub ahead of print].

10. Xing $D$, Kwong J, Yang $Z$, et al. Intra-articular injection of mesenchymal stem cells in treating knee osteoarthritis: a systematic review of animal studies. Osteoarthritis Cartilage 2018;26:445-61.

11. Kong L, Zheng LZ, Qin L, et al. Role of mesenchymal stem cells in osteoarthritis treatment. J Orthop Translat 2017;9:89-103.

12. Lee WY, Wang B. Cartilage repair by mesenchymal stem cells: clinical trial update and perspectives. J Orthop Translat 2017;9:76-88.

13. Nooshabadi VT, Mardpour S, Yousefi-Ahmadipour A, et al. The extracellular vesicles-derived from mesenchymal stromal cells: A new therapeutic option in regenerative medicine. J Cell Biochem. 2018;119:8048-73.

14. Cosenza S, Ruiz M, Toupet K, et al. Mesenchymal stem cells derived exosomes and microparticles protect cartilage and bone from degradation in osteoarthritis. Sci Rep 2017;7:16214.

15. Zhu H, Ji J, Fu T, et al. SAT0057 The effect of exosomes from bone marrow mesenchymal stem cells on osteoarthritis. Ann Rheum Dis 2018;77:893. 